\title{
Perinatal Vertical Transmission of Chikungunya Virus in Ruili, a Town on the Border between China and Myanmar
}

\author{
Jia-Yuan Shen ${ }^{1} \cdot$ Man $\mathrm{Li}^{1} \cdot$ Lyu Xie $^{1} \cdot$ Jia-Rong Mao ${ }^{2} \cdot$ Hong-Ning Zhou ${ }^{1,3} \cdot$ Pei-Gang Wang ${ }^{4}(\mathbb{D} \cdot$ \\ Jin-Yong Jiang ${ }^{1,3}$ (D) Jing $\mathrm{An}^{4}$ (D)
}

Received: 20 January 2020 / Accepted: 18 May 2020 / Published online: 16 July 2020

(C) The Author(s) 2020

\section{Dear Editor,}

Chikungunya virus (CHIKV), an arbovirus in the family of Togaviridae, genus Alphavirus, is transmitted by the $A$. aegyptii or A. albopictus mosquito, and causes disease in humans characterized by fever, rash, and arthralgia (Silva and Dermody 2017; Suhrbier 2019). It was first reported in 1953 in Tanzania, and caused only a few outbreaks and sporadic cases in Africa and Asia in last century. However, in the epidemic in 2004, CHIKV acquired mutations that conferred enhanced transmission by the A. albopictus mosquito (Schuffenecker et al. 2006). Since then, it has successively caused outbreaks in Africa, the Indian Ocean, South East Asia, the South America, and Europe (Zeller et al. 2016).

During the southern India Ocean epidemic in 2005, the vertical transmission of CHIKV during the perinatal period was observed in Reunion island (Lenglet et al. 2006). Of 160 pregnant women infected with CHIKV, 33 had viremia at the time of delivery (Lenglet et al. 2006). As a consequence, 16 neonates developed symptoms several days after their birth, and the risk of perinatal vertical transmission was about

Jia-Yuan Shen, Man Li and Lyu Xie contributed equally to this work.

Pei-Gang Wang

pgwang@ccmu.edu.cn

$\triangle$ Jin-Yong Jiang

yipdjiang@126.com

$\triangle$ Jing An

anjing@ccmu.edu.cn

1 Yunnan Institute of Parasitic Diseases, Simao Pu'er 665000, China

2 Ruili People's Hospital, Ruili 678600, China

3 Yunnan Provincial Key Laboratory of Vector-Borne Diseases Control and Research and Yunnan Provincial Collaborative Innovation Center for Public Health and Disease Prevention and Control (YPCICPHDPC), Simao Pu'er 665000, China

4 Department of Microbiology, School of Basic Medical Sciences, Capital Medical University, Beijing 100069, China
48.5\% (Lenglet et al. 2006). Two years later, as reported by a cohort study focusing on the neurocognitive function of children exposed to perinatal vertical transmission of CHIKV, $51 \%$ of 33 infected children had a global neurodevelopmental delay compared to $15 \%$ of 135 uninfected peers (Gerardin et al. 2014). These results indicated that perinatal vertical transmission of CHIKV resulted in a poor neurocognitive outcome (Gerardin et al. 2014). Thereafter, sporadic CHIKV infection through perinatal vertical transmission was observed in the Island of Curacao (van Enter et al. 2018), Brazil (Bandeira et al. 2016; Lyra et al. 2016), Colombia (Villamil-Gomez et al. 2015) and India (Kumar et al. 2019). In a retrospective study in Thailand and a cohort study in Colombia, however, no clinical findings suggestive of perinatal vertical transmission were observed (Laoprasopwattana et al. 2016; Escobar et al. 2017). So, how is CHIKV vertically transmitted during the perinatal period and what are the risk factors? More clinical observations are needed before these questions can be addressed.

Ruili of Yunnan Province is a town on the border between China and Myanmar (Fig. 1A). It has the circulation of both A. aegyptii and A. albopictus mosquitoes, and is threatened by various vector-borne infectious diseases. The Ruili People's Hospital is the largest hospital in Ruili, providing medical services to Chinese and neighboring Burmese. During September 2019, a CHIKV outbreak occurred in Ruili. By the end of December, more than 100 infected people were recorded according to the Direct Network Reporting Information System of Infectious Diseases in China. In this report, we describe two cases of perinatal vertical transmission in Ruili People's Hospital during the 2019 CHIKV epidemic. Three neonates born to a Burmese woman (twin pregnancy) and a Chinese woman, were vertically infected with CHIKV. They all had significant perinatal complications including rash, fever, and neonatal hyperbilirubinemia but didn't show obvious neurological symptoms. After treatment, they were all discharged from the hospital. 
A

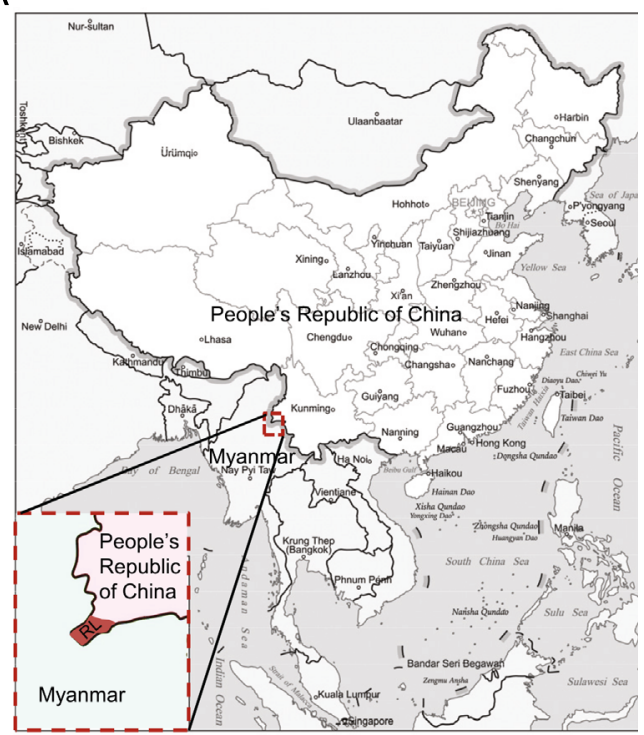

B

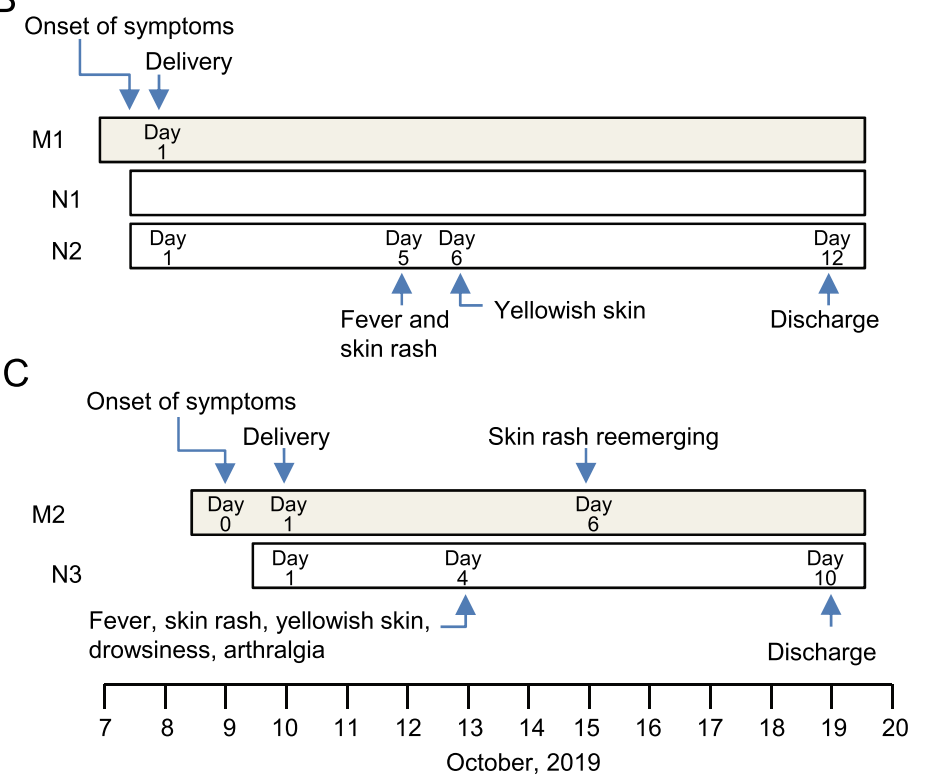

Fig. 1 Location of Ruili and timeline of two cases in this report. A Ruili of Yunnan Province locates on the border between China and Myanmar. B and $\mathbf{C}$ Timeline of two vertical transmission cases in Ruili People's Hospital. Three neonates (N1-N3) were infected with

Case 1

A 36-year-old Burmese woman (M1) was admitted to the obstetrical department of Ruili People's Hospital because of fever and myalgia for $10 \mathrm{~h}$ on October 8th, 2019. CHIKV infection was suspected as a CHIKV outbreak was ongoing in the city, and was validated later by RT-PCR. The same day she was admitted to hospital, she had a C-section delivery and gave birth to 2 male neonates ( $\mathrm{N} 1$ and $\mathrm{N} 2$ ).

The neonates were 36 weeks and 1 day gestational age, with birth weight of $2870 \mathrm{~g}$ (N1) and $2530 \mathrm{~g}$ (N2) respectively. They had neonatal asphyxia with Apgar scores of 6/6/9 (N1) and 6/6/8 (N2), respectively. They were immediately transferred to the neonatal intensive care unit (NICU) and placed on continuous positive airway pressure and later placed on BiPhasic non-invasive ventilator. Antibiotics (Cefperazone-Sulbactam $0.4 \mathrm{~g}$ q12h) were used to treat the presumable perinatal bacterial infection. On the fifth day of life, they developed disseminated maculopapular rash and fever with a peak of $39{ }^{\circ} \mathrm{C}$ and $38.9{ }^{\circ} \mathrm{C}$ respectively. Bathing with tepid water and taking acetaminophen orally didn't reduce the body temperature. One day later, yellowish skin appeared and the maximum serum indirect bilirubin reached $113.1 \mu \mathrm{mol} / \mathrm{L}$ (N1) and $100.6 \mu \mathrm{mol} / \mathrm{L}$ (N2) respectively. While in hospital, no convulsion, screaming, sleepiness and other neurological symptoms were observed. CHIKV infection was confirmed by RT-PCR. The twin progressed well and were discharged from hospital on the 12th day after delivery (Fig. 1B and Table 1).
CHIKV through perinatal vertical transmission from a Burmese woman (M1, twin pregnancy) and a Chinese women (M2). RL, Ruili City

\section{Case 2}

A 23-year-old Chinese woman (M2) developed fever, chest rash, and arthralgia on October 9th, 2019 at 38 weeks and 3 days of gestation. She was admitted to the obstetrical department of Ruili People's Hospital on Oct, 10th, and had a C-section delivery the same day. Her serum was positive for CHIKV IgM. CHIKV infection was further confirmed by RT-PCR. Six days after delivery, the skin rash reemerged.

The neonate (N3) was a female with birth weight of $3080 \mathrm{~g}$. Her Apgar score was 9/10/10, but the amniotic fluid was slightly stained. She was doing well until October 13th when she developed a macular erythematous rash, yellowish skin, drowsiness, arthralgia and temperature of $39{ }^{\circ} \mathrm{C}$. The maximum of blood indirect bilirubin was $160.8 \mathrm{mmol} / \mathrm{L}$ as recorded on Oct 14th. CHIKV infection was verified with RT-PCR. On the sixth day of her life, muscle tension was recovered but arthralgia was still present. No screaming, convulsion or other nervous system symptoms were observed while in hospital. After 6 days of antipyretic

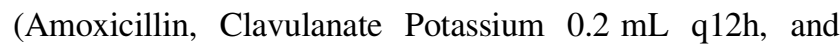
Cefperazone-Sulbactam $0.4 \mathrm{~g} \mathrm{q} 12 \mathrm{~h}$ ) and antiviral treatment (IFNB-1 $\alpha$ 800,000 iu bid), she progressed favorably and was discharged on her 10th day of life (Fig. 1C and Table 1).

Because dengue virus (DENV) is circulating in this region, all febrile patients should first be tested for DENV infection, and the negative cases will then be subjected to CHIKV or ZIKV detection by RT-PCR (Primers used for CHIKV RT-PCR: F: 5'- GGGCGGGTAGTCCATGTTGT 
Table 1 Characteristics of vertically infected neonates in Ruili People's Hospital

\begin{tabular}{llll}
\hline & M1 & M2 \\
\hline Age & 36 & 23 \\
Nationality & Myanmar & China \\
EGA at CHIKV infection (weeks + days) & $36+1$ & $38+3$ \\
EGA at delivery (weeks + days) & $36+1$ & $38+4$ \\
C-section delivery & Yes & Yes \\
& N1 & N2 & N3 \\
Sex & Male & Male & Female \\
Apgar score (1/5/10 min) & $6 / 6 / 9$ & $6 / 6 / 8$ & $9 / 10 / 10$ \\
Birthweight (gram) & 2870 & 2530 & 3080 \\
Amniotic fluid turbidity & No & No & Yes \\
Asphyxia neonatorum & Yes & Yes & No \\
First day of fever & 4 th & 4 th & 3 rd \\
Neonatal symptoms & & & \\
Fever & Yes & Yes & Yes \\
Irritability & No & No & No \\
Skin rash & Yes & Yes & Yes \\
Yellowish skin & Yes & Yes & Yes \\
Drowsiness & No & No & Yes \\
Indirect bilirubin ( $\mu$ mol/L) & 113.1 & 100.6 & 160.8 \\
Length in hospital (days) & 11 & 11 & 9 \\
Death & No & No & No \\
RT-PCR & + & + & + \\
\hline & & & \\
\hline
\end{tabular}

AGA-3'; R: 5'-ACCGGCGTCTACCCATTCATGT-3'). All

reported CHIKV cases in this study were negative for DENV and ZIKV.

According to a systematic review and meta-analysis on perinatal vertical transmission of CHIKV, the symptoms of infected neonates include fever, irritability, hyperalgesia, diffuse limb edema, rashes and occasionally sepsis-like illness and meningoencephalitis (Contopoulos-Ioannidis et al. 2018). Moreover, the manifestations are usually presented within the first week of life, but not at birth (Contopoulos-Ioannidis et al. 2018). In our report, the pregnant women had either neonatal asphyxia or amniotic fluid turbidity during delivery and C-section delivery was thus performed, indicating that the prenatal CHIKV infection increased the risk of pregnant women and neonates. Fever and rash were observed in all neonates, which presented on day 5 (case 1) and day 4 (case 2) respectively, keeping in line with previous reports (Contopoulos-Ioannidis et al. 2018). Besides fever and rash, all neonates exhibited neonatal hyperbilirubinemia. Hyperbilirubinemia is a higher-than-normal level of bilirubin in the blood. Neonatal hyperbilirubinemia is usually manifested as neonatal jaundice, which is a yellowish discoloration in the eyes and skin of a newborn baby due to high bilirubin levels. In many cases there is no specific underlying disorder (physiologic). In other cases, it results from red blood cell breakdown, liver disease, infection, hypothyroidism, or metabolic disorders (pathologic).
Hyperbilirubinemia during CHIKV infection has not been reported before, so that the underlying mechanism and its association with CHIKV infection need further investigation. None of the neonates had obvious neurological symptoms. However, as more than half of vertically infected children during perinatal period have been shown to have a global neurodevelopmental delay (Gerardin et al. 2014), appropriate follow-up is necessary to evaluate the long-term impact of CHIKV infection on neurodevelopment in this region.

These are the first cases of perinatal vertical transmission of CHIKV recorded in China. In 2010, China had a small epidemic of CHIKV in Guangdong province (Wu et al. 2013), which was followed by a few imported or sporadic cases in various regions in recent years. The circulation of CHIKV in Yunnan Province has been reviewed by Xia et al. (2018). The first imported sporadic CHIKV infection was reported in Xishuangbanna, Yunnan, in 1987, and the first isolation of CHIKV in China was taken from serum of a patient at that time (Xia et al. 2018). In 2001-2004, two serological investigations were carried out in Yunnan Province and the results showed that 2.63\%-11.03\% health people were positive for CHIKV antibody in the Lower Reaches Area of Lancang River in Yunnan Province (Wang et al. 2013). The circulation of CHIKV in border region between China and Myanmar, especially the detection of perinatal vertical transmission in the region is of significant concern. Research on the prevalence and prevention of CHIKV needs to be strengthened in China.

Since September 2019, more than 100 people have been infected with CHIKV according to the Direct Network Reporting Information System of Infectious Diseases in China. Except two women in our report, no other pregnant women were infected by CHIKV during the intrapartum period. Thus, in the three neonates born to two women with intrapartum infection, the risk of perinatal vertical transmission is $100 \%$, which is similar to that observed in Reunion island, Brazil, Colombia and India (Lenglet et al. 2006; Villamil-Gomez et al. 2015; Bandeira et al. 2016; Lyra et al. 2016; Kumar et al. 2019). In contrast, of 15 women delivered during acute CHIKV infection, as reported in 2015 in Colombia, 12 neonates were hospitalized to rule out vertical transmission, though no clinical findings suggestive of neonatal CHIKV infection were observed (Escobar et al. 2017). A similar result was also reported in Thailand in 2008 (Laoprasopwattana et al. 2016). It is still unknown why the rate of perinatal vertical transmission varied among these countries. During the epidemic in Reunion island, CHIKV acquired an A226V mutation in the envelop protein, which enhanced viral replication in A. albopictus mosquito (Schuffenecker et al. 2006). Thus, the difference of virus strain or the difference in mosquito might account for the discrepancy in these reports. To identify the factors determining perinatal vertical transmission of CHIKV, more genetic and animal studies are needed. 
Thousands of neonates born with microcephaly during the epidemic of Zika virus (ZIKV) raised concerns on mother-tochild transmission of vector-borne viruses. The observation of perinatal vertical transmission of CHIKV suggested attention should be paid not only to ZIKV. Unlike ZIKV whose vertical transmission occurs during the first trimester, CHIKV is usually transmitted from mother to child at the perinatal period (Contopoulos-Ioannidis et al. 2018). The risk of neonatal infections was $50 \%$ among intrapartum maternal infections vs $0 \%$ among antepartum/peripartum maternal infections (Contopoulos-Ioannidis et al. 2018). Although the mechanism underlying perinatal vertical transmission of CHIKV is not clear, the time point makes it impossible to terminate pregnancy, so its impact could be greater than ZIKV. More attention is needed for the long-term impact of CHIKV on the health of newborns, and more efforts are required to clarify the mechanisms of pathogenesis to propose effective strategies to prevent neonatal CHIKV infection.

Acknowledgements This study was supported by the National Natural Science Foundation of China under grants U1602223, 81671971, 81972979, 81772172, and 81871641; the Scientific Research Plan of the Beijing Municipal Education Committee under grant KM201710025002; the Key Project of Beijing Natural Science Foundation B under grant KZ201810025035; the Yunnan Institute of Parasitic Diseases Innovative Team of Key Techniques for Vector Borne Disease Control and Prevention (Developing) under grant 2019HC010; and the Academician Workstation of Professor Jin Ningyi \& Training Base of International Scientific Exchange and Education in Tropical Diseases for South and Southeast Asia under grant 2018IC151.

\section{Compliance with Ethical Standards}

Conflict of Interest The authors declare that they have no conflicts of interest.

Animal and Human Rights Statement The research protocol was approved by the Ethics Committee of Yunnan Institute of Parasitic Diseases. Written informed consent was obtained from all participants prior to sample collection.

Open Access This article is licensed under a Creative Commons Attribution 4.0 International License (https://creativecommons.org/ licenses/by/4.0/), which permits use, sharing, adaptation, distribution and reproduction in any medium or format, as long as you give appropriate credit to the original author(s) and the source, provide a link to the Creative Commons licence, and indicate if changes were made. The images or other third party material in this article are included in the article's Creative Commons licence, unless indicated otherwise in a credit line to the material. If material is not included in the article's Creative Commons licence and your intended use is not permitted by statutory regulation or exceeds the permitted use, you will need to obtain permission directly from the copyright holder. To view a copy of this licence, visit http://creativecommons.org/licenses/by/4.0/.

\section{References}

Bandeira AC, Campos GS, Sardi SI, Rocha VF, Rocha GC (2016) Neonatal encephalitis due to Chikungunya vertical transmission: First report in Brazil. IDCases 5:57-59
Contopoulos-Ioannidis D, Newman-Lindsay S, Chow C, LaBeaud AD (2018) Mother-to-child transmission of Chikungunya virus: A systematic review and meta-analysis. PLoS Negl Trop Dis 12:e0006510

Escobar M, Nieto AJ, Loaiza-Osorio S, Barona JS, Rosso F (2017) Pregnant Women Hospitalized with Chikungunya Virus Infection, Colombia, 2015. Emerg Infect Dis 23

Gerardin P, Samperiz S, Ramful D, Boumahni B, Bintner M, Alessandri JL, Carbonnier M, Tiran-Rajaoefera I, Beullier G, Boya I, Noormahomed T, Okoi J, Rollot O, Cotte L, Jaffar-Bandjee MC, Michault A, Favier F, Kaminski M, Fourmaintraux A, Fritel X (2014) Neurocognitive outcome of children exposed to perinatal mother-to-child Chikungunya virus infection: the CHIMERE cohort study on Reunion Island. PLoS Negl Trop Dis 8:e2996

Kumar S, Agrawal G, Wazir S, Kumar A, Dubey S, Balde M, Yadav BS (2019) Experience of Perinatal and Neonatal Chikungunya Virus (CHIKV) Infection in a Tertiary Care Neonatal Centre during Outbreak in North India in 2016: A Case Series. J Trop Pediatr 65:169-175

Laoprasopwattana K, Suntharasaj T, Petmanee P, Suddeaugrai O, Geater A (2016) Chikungunya and dengue virus infections during pregnancy: seroprevalence, seroincidence and maternalfetal transmission, southern Thailand, 2009-2010. Epidemiol Infect 144:381-388

Lenglet Y, Barau G, Robillard PY, Randrianaivo H, Michault A, Bouveret A, Gerardin P, Boumahni B, Touret Y, Kauffmann E, Schuffenecker I, Gabriele M, Fourmaintraux A (2006) Chikungunya infection in pregnancy: Evidence for intrauterine infection in pregnant women and vertical transmission in the parturient. Survey of the Reunion Island outbreak. J Gynecol Obstet Biol Reprod (Paris) 35:578-583

Lyra PP, Campos GS, Bandeira ID, Sardi SI, Costa LF, Santos FR, Ribeiro CA, Jardim AM, Santiago AC, de Oliveira PM, Moreira LM (2016) Congenital Chikungunya Virus Infection after an Outbreak in Salvador, Bahia, Brazil. AJP Rep 6:e299-300

Schuffenecker I, Iteman I, Michault A, Murri S, Frangeul L, Vaney MC, Lavenir R, Pardigon N, Reynes JM, Pettinelli F, Biscornet L, Diancourt L, Michel S, Duquerroy S, Guigon G, Frenkiel MP, Brehin AC, Cubito N, Despres P, Kunst F, Rey FA, Zeller H, Brisse $S$ (2006) Genome microevolution of chikungunya viruses causing the Indian Ocean outbreak. PLoS Med 3:e263

Silva LA, Dermody TS (2017) Chikungunya virus: epidemiology, replication, disease mechanisms, and prospective intervention strategies. J Clin Invest 127:737-749

Suhrbier A (2019) Rheumatic manifestations of chikungunya: emerging concepts and interventions. Nat Rev Rheumatol 15:597-611

van Enter BJD, Huibers MHW, van Rooij L, Steingrover R, van Hensbroek MB, Voigt RR, Hol J (2018) Perinatal Outcomes in Vertically Infected Neonates During a Chikungunya Outbreak on the Island of Curacao. Am J Trop Med Hyg 99:1415-1418

Villamil-Gomez W, Alba-Silvera L, Menco-Ramos A, GonzalezVergara A, Molinares-Palacios T, Barrios-Corrales M, Rodriguez-Morales AJ (2015) Congenital Chikungunya Virus Infection in Sincelejo, Colombia: A Case Series. J Trop Pediatr 61:386-392

Wang Y-H, Bao F-K, Liu A-H (2013) Current progress in research of Chikungunya fever in Yunnan Province in China. China Trop Med 13:4

Wu D, Zhang Y, Zhouhui Q, Kou J, Liang W, Zhang H, Monagin C, Zhang Q, Li W, Zhong H, He J, Li H, Cai S, Ke C, Lin J (2013) Chikungunya virus with E1-A226V mutation causing two outbreaks in 2010, Guangdong. China Virol J 10:174

Xia H, Wang Y, Atoni E, Zhang B, Yuan Z (2018) MosquitoAssociated Viruses in China. Virol Sin 33:5-20

Zeller H, Van Bortel W, Sudre B (2016) Chikungunya: Its History in Africa and Asia and Its Spread to New Regions in 2013-2014. J Infect Dis 214:S436-S440 